BMJ Open

Diabetes

Research

\& Care

\title{
Cardiac structure and function with and without metabolic syndrome: the Echocardiographic Study of Latinos (Echo-SOL)
}

\author{
Melissa Burroughs Peña, ${ }^{1}$ Katrina Swett, ${ }^{2}$ Neil Schneiderman, ${ }^{3}$ Daniel M Spevack, ${ }^{4}$ \\ Sonia G Ponce, ${ }^{5}$ Gregory A Talavera, ${ }^{6}$ Mayank M Kansal, ${ }^{7}$ Martha L Daviglus, ${ }^{8}$
} Jianwen Cai, ${ }^{9}$ Barry E Hurwitz, ${ }^{3}$ Maria M Llabre, ${ }^{3}$ Carlos J Rodriguez ${ }^{2}$

\begin{abstract}
To cite: Burroughs Peña M, Swett K, Schneiderman N, et al. Cardiac structure and function with and without metabolic syndrome: the Echocardiographic Study of Latinos (Echo-SOL). BMJ Open Diab Res Care 2018;6:e000484. doi:10.1136/ bmjdrc-2017-000484
\end{abstract}

Received 19 0ctober 2017 Revised 11 April 2018 Accepted 19 April 2018

Check for updates

(c) Author(s) (or their employer(s)) 2018. Re-use permitted under CC BY-NC. No commercial re-use. See rights and permissions. Published by BMJ.

For numbered affiliations see end of article.

Correspondence to Dr Melissa Burroughs Peña; mburroughspena@ stanfordhealthcare.org

\section{ABSTRACT}

Objective We assessed the hypothesis that metabolic syndrome is associated with adverse changes in cardiac structure and function in participants of the Echocardiographic Study of Latinos (Echo-SOL). Methods Non-diabetic Echo-SOL participants were included in this cross-sectional analysis. Metabolic syndrome was defined according to the American Heart Association/ National Heart, Lung, and Blood Institute 2009 Joint Scientific Statement. Survey multivariable linear regression analyses using sampling weights were used adjusting for multiple potential confounding variables. Additional analysis was stratified according to the presence/absence of obesity (body mass index $(\mathrm{BMI}) \geq 25 \mathrm{~kg} / \mathrm{m}^{2}$ ) and the presence/absence of metabolic syndrome.

Results Within Echo-SOL, 1260 individuals met inclusion criteria ( $59 \%$ female; mean age 55.2 years). Compared with individuals without metabolic syndrome, those with metabolic syndrome had lower medial and lateral $\mathrm{E}^{\prime}$ velocities $(-0.4 \mathrm{~cm} / \mathrm{s},(\mathrm{SE} 0.1), \mathrm{p}=0.0002 ;-0.5 \mathrm{~cm} / \mathrm{s}(0.2), \mathrm{p}=0.02$, respectively), greater $E / E^{\prime}(0.5(0.2), p=0.01)$ and worse two-chamber left ventricular longitudinal strain $(0.9 \%(0.3)$, $p=0.009$ ), after adjusting for potential confounding variables. Increased left ventricular mass index $\left(9.8 \mathrm{~g} / \mathrm{m}^{2}(1.9)\right.$, $p<0.0001$ and $\left.7.5 \mathrm{~g} / \mathrm{m}^{2}(1.7), p<0.0001\right)$, left ventricular enddiastolic volume (11.1 mL (3.0), $p=0.0003$ and $13.3 \mathrm{~mL}$ (2.7), $p<0.0001)$, left ventricular end-systolic volume $(5.0 \mathrm{~mL}(1.4)$, $p=0.0004$ and $5.7 \mathrm{~mL}(1.3) p<0.0001)$ and left ventricular stroke volume (10.2 mL (1.8), $p<0.0001$ and $13.0 \mathrm{~mL}(2.0)$, $\mathrm{p}<0.0001$ ) were observed in obese individuals with and without metabolic syndrome compared with individuals with normal weight without metabolic syndrome. In sensitivity analyses, individuals with normal weight $\left(\mathrm{BMl}<25 \mathrm{~kg} / \mathrm{m}^{2}\right)$ and metabolic syndrome had worse left ventricular global longitudinal strain $(2.1 \%(0.7), p=0.002)$ and reduced left ventricular ejection fraction $(-3.5 \%(1.4), p=0.007)$ compared with normal-weight individuals without metabolic syndrome.

Conclusions In a sample of US Hispanics/Latinos metabolic syndrome was associated with worse left ventricular systolic and diastolic function. Adverse changes in left ventricular size and function were observed in obese individuals with and without metabolic syndrome but decreased left ventricular function was also present in normal-weight individuals with metabolic syndrome.

\section{Significance of this study}

What is already known about this subject?

- Metabolic syndrome is prevalent in $35 \%$ of Hispanics/Latinos in the USA.

What are the new findings?

- In Hispanics/Latinos metabolic syndrome is associated with decreased left ventricular diastolic and systolic function, even in individuals with normal body mass index.

How might these results change the focus of research or clinical practice?

- Hispanic/Latino patients with metabolic syndrome might represent an at-risk population to target interventions for primordial prevention of heart failure.

\section{INTRODUCTION}

Hispanics/Latinos are the largest ethnic minority group in the USA, comprising $17 \%$ of the population ( 55 million) in $2014 .{ }^{1}$ While Hispanics/Latinos represent diverse racial and cultural backgrounds from across the Americas, several alarming trends in cardiovascular health have emerged in this population. Compared with non-Hispanic whites, Hispanics/Latinos have a higher prevalence of obesity, diabetes, and dyslipidemia. ${ }^{2}{ }^{3}$ Additionally, Hispanics/Latinos have a high prevalence of metabolic syndrome, demonstrating poor metabolic health across several measures. ${ }^{45}$ In the population-based cohort Hispanic Community Health Study/Study of Latinos (HCHS/SOL), $35 \%$ of participants met the criteria for metabolic syndrome. However, the long-term impact of metabolic syndrome on cardiovascular outcomes in Hispanics/Latinos is not well understood.

Metabolic syndrome is associated with multiple adverse cardiovascular outcomes including heart failure, coronary heart disease, 
and cardiovascular mortality. ${ }^{6-9}$ Incident heart failure in normal-weight individuals with metabolic syndrome is in fact greater than incident heart failure in overweight individuals without the metabolic syndrome. ${ }^{10}$ Yet, the relationship between metabolic syndrome and heart failure risk in Hispanics/Latinos has not been well described. Hispanics/Latinos in the USA have a higher incidence of heart failure compared with non-Hispanic whites, and Hispanics/Latinos who present with heart failure are younger with more comorbidities and lower left ventricular ejection fraction. ${ }^{11-13}$ However, Hispanics/Latinos in the USA represent a relatively young demographic such that the future public health burden of clinical heart failure as this population ages is potentially underestimated. In order to identify preclinical disease, abnormalities in cardiac structure and function can be determined by echocardiography and cardiac MRI, many of which have been associated with incident cardiovascular disease and increased mortality including asymptomatic left ventricular systolic and diastolic dysfunction, left ventricular hypertrophy, left ventricular size in diastole and left atrial size. ${ }^{14-19}$ Recent data suggest that even borderline left ventricular systolic function is associated with incident heart failure and increased all-cause mortality. ${ }^{20}$ Therefore, understanding the complex relationship of cardiometabolic risk factors and body mass index with cardiac structure and function offers an opportunity to risk stratify individuals and prevent future adverse cardiovascular outcomes.

The primary objective of this study was to determine the associations of metabolic syndrome and its individual criteria with cardiac structure and function as measured by transthoracic echocardiography in participants in the Echocardiographic Study of Latinos (Echo-SOL). Our secondary objective was to compare cardiac structure and function in Echo-SOL participants according to normal/ abnormal body mass index (BMI) and prevalent metabolic syndrome.

\section{METHODS}

Study setting

HCHS/SOL is a population-based longitudinal cohort study ( $\mathrm{n}=16415$, mean age 43) of self-identified Hispanic/ Latino individuals, $18-74$ years of age. ${ }^{21}$ This study was designed to assess chronic disease in Hispanic/Latino individuals living in four cities in the USA: Bronx, New York; Chicago, Illinois; Miami, Florida; and San Diego, California. Participants were recruited in communities surrounding four field centers in the respective cities. The details of HCHS/SOL sampling method have been previously described. ${ }^{21}$ Exclusion criteria included: activeduty military service, not living at the residential address, planning to move from the area within 6 months, or physically unable to attend the clinic examination. The baseline HCHS/SOL assessment was completed from 2008 to 2011 and consisted of a questionnaire, anthropometric measurements, and laboratory testing.
Echo-SOL is an ancillary study of HCHS/SOL consisting of 1824 participants recruited through a stratified-sampling process representative of the parent study. ${ }^{22}$ Echo-SOL was designed to characterize cardiac structure and function using transthoracic echocardiography in a representative sample of Hispanic/Latino individuals living in the USA. The baseline echocardiography examination for the Echo-SOL ancillary study was performed from 2011 to $2014 .^{22}$ Inclusion criteria for Echo-SOL included: age 45 years or older; self-reported Hispanic/ Latino of Mexican, Puerto Rican, Cuban, Dominican, Central American or South American background; and enrollment 36 months or fewer from the date of the baseline HCHS/SOL visit. Echo-SOL enrolled on average $\sim 80 \%$ of eligible participants at each of the study sites.

In this cross-sectional study of the Echo-SOL cohort, we included non-diabetic participants without coronary artery disease or severe aortic or mitral valve disease. Coronary artery disease was determined by self-report or pathologic Q-waves on ECG consistent with prior myocardial infarction. Aortic and mitral valve disease was determined by transthoracic echocardiography.

\section{Study procedures}

Phillips IE-33 or Sonos 5500/7500 ultrasound imaging platforms with a standard 2.5 to $3.5 \mathrm{MHz}$ phased-array probe were used in the acquisition of all echocardiographic imaging data. A standard transthoracic echocardiograpy examination was performed with the participant in the partial left decubitus position. ECG-gated M-mode, 2D, spectral, color flow, and tissue Doppler images were acquired in the parasternal long axis, short axis, and apical four-chamber and two-chamber long-axis views. Left ventricular global longitudinal strain analysis was performed using the vendor-independent TomTec Cardiac Performance Analysis software (TomTec, Hamden, Connecticut, USA) on acquired 2D images. All images were read by a sonographer and over-read by a cardiologist (CJR) with level 3 echocardiography training as specified by the American College of Cardiology and the American Society of Echocardiography ${ }^{23}$ Inter-reader and intrareader variability were assessed and determined to have a high degree of intraclass correlation for each measurement (0.80-0.99).

\section{Definitions}

In this study, we used the American Heart Association/ National Heart, Lung, and Blood Institute ${ }^{3}$ Joint Scientific Statement criteria to define metabolic syndrome as three or more of the following: fasting glucose $\geq 100 \mathrm{mg} / \mathrm{dL}$ and/or on antihyperglycemic medication; triglycerides $\geq 150 \mathrm{mg} / \mathrm{dL}$ and/or on triglyceride-lowering medication; $\mathrm{HDL}<40 \mathrm{mg} / \mathrm{dL}$ in men and $<50 \mathrm{mg}$ / $\mathrm{dL}$ in women and/or on HDL-raising medication; waist circumference $\geq 102 \mathrm{~cm}$ in men and $\geq 88 \mathrm{~cm}$ in women; systemic systolic blood pressure $\geq 130 \mathrm{~mm} \mathrm{Hg}$, systemic diastolic blood pressure $\geq 85 \mathrm{~mm} \mathrm{Hg}$, and/or on antihypertensive medication. 
The echocardiographic measurements included in this study included multiple measures of left and right heart structure and function: left ventricular mass indexed to body surface area (LVMI), left ventricular end-diastolic volume (LVEDV), left ventricular end-systolic volume (LVESV), left ventricular ejection fraction (LVEF), left ventricular stroke volume, medial and lateral tissue Doppler $\mathrm{E}^{\prime}$ velocities, mitral inflow $\mathrm{E}$ velocity and $\mathrm{A}$ velocity ratio $(\mathrm{E} / \mathrm{A})$, mitral valve $\mathrm{E}$ velocity and $\mathrm{E}^{\prime}$ velocity ratio $\left(\mathrm{E} / \mathrm{E}^{\prime}\right)$, isovolumic relaxation time (IVRT), peak right ventricular/right atrial pressure gradient, tricuspid annular plane systolic excursion (TAPSE), right ventricular fractional area change, left atrial volume index, left ventricular global longitudinal strain (GLS) (individual four-chamber and two-chamber views and average).

Echo-SOL participant sociodemographic and lifestyle characteristics were obtained by questionnaires conducted during the HCHS/SOL baseline visit. National background was determined by self-report and classified as: Mexican, Puerto Rican, Cuban, Dominican, Central American, or South American. Alcohol and tobacco use was determined by self-report and characterized as current, former, or never. Education was described by three categories: less than high school, high school or equivalent, or greater than high school. Household income was categorized into five groups: <US\$20 000, US $\$ 20001-40$ 000, US $\$ 40001-75000$, or >US $\$ 75000$. US born was defined as born in the 50 states of USA, excluding US territories. Physical activity was determined using the Global Physical Activity Questionnaire and was classified as low, moderate, or high.

\section{Statistical methods}

The primary aim of this study was to compare echocardiographic measures of cardiac structure and function in participants with and without metabolic syndrome. The baseline characteristics of participants with and without metabolic syndrome were compared. The corresponding distribution of all baseline sociodemographic and clinical characteristics was summarized for the overall population using means $\pm \mathrm{SE}$ for continuous variables and proportions for categorical variables. Continuous variables were compared using elementwise regression using generalized least squares estimation. This methodology was chosen due to the sampling weighting to account for survey methods and nonresponse. Categorical variables were compared using Rao-Scott $\mathrm{X}^{2}$. The mean values of the echocardiographic outcome variables in participants with and without metabolic syndrome were compared using t-tests. Summary statistics were weighted to adjust for sampling probability and non-response. Multivariable linear regression analyses were used to model the relationships between metabolic syndrome and each echocardiographic outcome variable described above adjusting for the following covariates: Minimally adjusted model (age and sex) and fully adjusted model (age, sex, study site, tobacco use, alcohol use, physical activity, and education).
A priori multivariable linear regression analyses were conducted to model the relationship between the individual components of metabolic syndrome (ie, fasting glucose $\geq 100 \mathrm{mg} / \mathrm{dL}$ ) with the following echocardiographic measurements: LVMI, LVEDV, LV GLS, and $\mathrm{E} / \mathrm{E}^{\prime}$. Regression models were adjusted for covariates as above.

The secondary aim of this study was to compare cardiac structure and function in participants with and without metabolic syndrome by BMI. Multivariable linear regression analyses were stratified by BMI comparing individuals with and without metabolic syndrome in the following strata: normal weight, overweight and obese. The models adjusted for the following covariates: age, sex, site, tobacco use, alcohol use, years of education, and physical activity. Sensitivity analyses were performed using linear regression analyses of the aggregate data with interaction terms to evaluate for interaction between BMI and metabolic syndrome for each echocardiographic measure of structure and function. In sensitivity analyses, multivariable linear regression analyses were used to compare the echocardiographic measurements of cardiac structure and function in normal weight (BMI $<25 \mathrm{~kg} / \mathrm{m}^{2}$ ) participants without metabolic syndrome to the following groups: obese (BMI $\geq 30 \mathrm{~kg} / \mathrm{m}^{2}$ ) without metabolic syndrome; obese (BMI $\geq 30 \mathrm{~kg} / \mathrm{m}^{2}$ ) with metabolic syndrome; overweight (BMI $\geq 25 \mathrm{~kg} / \mathrm{m}^{2}$ and $<30 \mathrm{~kg}$ / $\mathrm{m}^{2}$ ) without metabolic syndrome; overweight (BMI $\geq 25$ $\mathrm{kg} / \mathrm{m}^{2}$ and $<30 \mathrm{~kg} / \mathrm{m}^{2}$ ) with metabolic syndrome; and all weights with metabolic syndrome. The model adjusted for the following covariates: age, sex, site, tobacco use, alcohol use, years of education, and physical activity. All statistical analyses were performed with SAS software V.9.3 and weighted to adjust for sampling probability and non-response.

\section{RESULTS}

Within the Echo-SOL cohort 1260 non-diabetic participants (mean age 55.2 years) who met the inclusion criteria were included in this analysis, with 399 (32\%) participants meeting the criteria for metabolic syndrome. The baseline characteristics of the participants with and without metabolic syndrome are described in table 1. Compared with those without metabolic syndrome, participants with metabolic syndrome were older with a greater proportion of participants between 55 and 64 years of age. Participants with metabolic syndrome also tended to be Cuban or Puerto Rican and to live in Miami. Individuals with metabolic syndrome had a greater prevalence of low physical activity and lower education and income. Mean BMI was higher in individuals with metabolic syndrome $\left(32 \mathrm{~kg} / \mathrm{m}^{2}\right)$ but was also elevated in individuals without metabolic syndrome $\left(29 \mathrm{~kg} / \mathrm{m}^{2}\right)$. The distribution of individuals with normal weight, overweight and obesity with and without metabolic syndrome are presented in online supplementary figure 1. 
Table 1 Characteristics of Echocardiographic Study of Latinos participants with and without metabolic syndrome

Metabolic syndrome,

Without metabolic syndrome, $\mathrm{n}=399$

$\mathrm{n}=861$

$P$ values

\begin{tabular}{|c|c|c|c|}
\hline \multicolumn{4}{|l|}{ Age, mean (SE) } \\
\hline$<55$ & $46.4(4.6)$ & $57.6(2.7)$ & \multirow[t]{3}{*}{0.11} \\
\hline $55-64$ & $36.1(3.7)$ & $26.1(2.1)$ & \\
\hline $65+$ & $17.5(3.4)$ & $16.3(3.2)$ & \\
\hline Female gender, $\mathrm{N}(\%)$ & $274(59.8)$ & $568(58.1)$ & 0.74 \\
\hline \multicolumn{4}{|l|}{ National background, N (\%) } \\
\hline Dominican & 64 (14.7) & 160 (19.2) & \multirow[t]{6}{*}{0.08} \\
\hline Puerto Rican & $72(19.5)$ & $145(15.5)$ & \\
\hline Mexican & $95(16.8)$ & $227(23.5)$ & \\
\hline Cuban & $99(38.7)$ & $158(28.8)$ & \\
\hline Central American & $49(6.3)$ & $78(5.8)$ & \\
\hline South American & $20(4.1)$ & $90(7.2)$ & \\
\hline \multicolumn{4}{|l|}{ Site, N (\%) } \\
\hline Bronx & $106(31.1)$ & $251(32.7)$ & \multirow[t]{4}{*}{0.08} \\
\hline Chicago & $91(10.1)$ & $199(11.7)$ & \\
\hline Miami & $134(44.9)$ & $232(35.0)$ & \\
\hline San Diego & $64(13.9)$ & $179(20.7)$ & \\
\hline Low physical activity, N (\%) & $276(70.8)$ & $543(60.5)$ & 0.04 \\
\hline \multicolumn{4}{|l|}{ Tobacco use, N (\%) } \\
\hline Current & $63(16.4)$ & $148(18.5)$ & \multirow[t]{2}{*}{0.25} \\
\hline Former & $106(29.0)$ & $183(22.5)$ & \\
\hline \multicolumn{4}{|l|}{ Years of education, N (\%) } \\
\hline Less than high school & $142(32.5)$ & $261(30.1)$ & \multirow[t]{3}{*}{0.36} \\
\hline High school & $96(24.8)$ & $190(20.2)$ & \\
\hline Greater than high school & $152(42.7)$ & $387(49.6)$ & \\
\hline Household income <US\$20 000/year, N (\%) & $196(59.7)$ & $389(46.1)$ & 0.006 \\
\hline US mainland born, $\mathrm{N}(\%)$ & $35(7.4)$ & $85(9.6)$ & 0.41 \\
\hline Body mass index, $\mathrm{kg} / \mathrm{m}^{2}$ (SE) & $31.5[0.3]$ & $28.5(0.4)$ & $<0.0001$ \\
\hline Fasting glucose, mg/dL (SE) & $98.2(1.0)$ & $93.0(0.3)$ & $<0.0001$ \\
\hline High-density lipoprotein, mg/dL (SE) & $44.1(0.7)$ & $55.1(0.5)$ & $<0.0001$ \\
\hline Triglyceride, mg/dL (SE) & $184.5(5.2)$ & $133.4(1.3)$ & $<0.0001$ \\
\hline Waist circumference, cm (SE) & $103.3(0.8)$ & $94.7(0.5)$ & $<0.0001$ \\
\hline Systolic blood pressure, mm Hg (SE) & $133.2(1.3)$ & $125.5(0.8)$ & $<0.0001$ \\
\hline Diastolic blood pressure, mm Hg (SE) & $78.4(0.7)$ & $74.4(0.4)$ & $<0.0001$ \\
\hline Antihypertensive medication use, N (\%) & $117(26.8)$ & $91(14.6)$ & 0.003 \\
\hline Antihyperglycemic medication use, N (\%) & $3(0.64)$ & $2(0.08)$ & 0.03 \\
\hline Lipid-lowering medication use, $\mathrm{N}(\%)$ & $48(17.7)$ & 87 (10.2) & 0.10 \\
\hline
\end{tabular}

Rao-Scott $\mathrm{X}^{2}$ test used to compare categorical variables in individuals with and without metabolic syndrome. Elementwise regression using generalized least squares estimation was used to compare continuous variables in individuals with and without metabolic syndrome.

Percentages are weighted to account for sampling and non-response.

Table 2 shows the mean values of each echocardiographic measurement in individuals with and without metabolic syndrome. Of note, the mean values for several measures of diastolic dysfunction including medial and lateral $\mathrm{E}^{\prime}$ velocities, $\mathrm{E} / \mathrm{E}^{\prime}$ ratio, and isovolumic relaxation time were abnormal in individuals with and without metabolic syndrome. The mean values of echocardiographic measures of right and left ventricular systolic function were normal in both groups. 
Table 2 Mean values of echocardiographic measures in Echo-SOL participants with and without metabolic syndrome

\begin{tabular}{|c|c|c|c|}
\hline & $\begin{array}{l}\text { Metabolic } \\
\text { syndrome (SE) }\end{array}$ & $\begin{array}{l}\text { Without metabolic } \\
\text { syndrome (SE) }\end{array}$ & $P$ values \\
\hline LV mass index, $\mathrm{g} / \mathrm{m}^{2}$ & $81.3(1.0)(n=389)$ & $79.2(1.0)(n=845)$ & 0.14 \\
\hline LV end-diastolic volume, $\mathrm{mL}$ & $84.0(1.7)(n=396)$ & $83.8(1.0)(n=854)$ & 0.92 \\
\hline LV end-systolic volume, mL & $33.5(0.8)(n=395)$ & $33.5(0.5)(n=854)$ & 0.98 \\
\hline LV ejection fraction, \% & $60.4(0.3)(n=377)$ & $60.2(0.2)(n=836)$ & 0.73 \\
\hline LV stroke volume, mL & $70.1(1.4)(n=393)$ & $70.6(0.9)(n=848)$ & 0.76 \\
\hline Medial E' velocity, cm/s & $6.8(0.1)(n=395)$ & $7.5(0.1)(n=856)$ & $<0.0001$ \\
\hline Lateral E' velocity, $\mathrm{cm} / \mathrm{s}$ & $9.0(0.2)(n=394)$ & $9.9(0.2)(n=849)$ & 0.002 \\
\hline E/A ratio & $1.0(0.02)(n=395)$ & $1.1(0.01)(n=848)$ & 0.0003 \\
\hline$E / E^{\prime}$ ratio & $10.0(0.2)(n=394)$ & $9.3(0.2)(n=848)$ & 0.0051 \\
\hline Isovolumic relaxation time, s & $0.1(0.001)(n=394)$ & $0.1(0.0009)(n=850)$ & 0.44 \\
\hline Peak RV/RA pressure gradient, $\mathrm{mm} \mathrm{Hg}$ & $17.6(0.6)(n=373)$ & $17.8(0.3)(n=821)$ & 0.76 \\
\hline TAPSE, cm & $2.1(0.03)(n=377)$ & $2.1(0.03)(n=827)$ & 0.85 \\
\hline $\mathrm{RV}$ fractional area change, $\%$ & $0.5(0.006)(n=365)$ & $0.5(0.006)(n=797)$ & 0.24 \\
\hline Left atrial volume index, $\mathrm{mL} / \mathrm{m}^{2}$ & $22.7(0.5)(n=365)$ & $23.2(0.03)(n=845)$ & 0.36 \\
\hline LV longitudinal strain (four chamber), \% & $-18.9(0.4)(n=391)$ & $-18.7(0.2)(n=846)$ & 0.61 \\
\hline LV longitudinal strain (two chamber), \% & $-19.5(0.3)(n=379)$ & $-20.3(0.3)(n=829)$ & 0.027 \\
\hline LV global longitudinal strain (average), \% & $-19.0(0.3)(n=391)$ & $-19.5(0.2)(n=845)$ & 0.12 \\
\hline
\end{tabular}

$E / A$, mitral inflow $E$ velocity and $A$ velocity ratio; $E / E^{\prime}$, mitral valve $E$ velocity and $E^{\prime}$ velocity ratio; Echo-Sol, Echocardiographic Study of Latinos; LV, left ventricle; RA, right atrium; RV, right ventricle; TAPSE, tricuspid annular plane systolic excursion.

Multivariable linear regression analyses of the relationship between metabolic syndrome with measurements of cardiac structure and function were notable for worse left ventricular diastolic function and systolic function in individuals with metabolic syndrome compared with individuals without metabolic syndrome (table 3). Specifically, in the fully adjusted models, individuals with metabolic syndrome had lower medial $\mathrm{E}^{\prime}$ velocity $(-0.4 \mathrm{~cm} / \mathrm{s}$ (SE 0.1)), lower lateral $\mathrm{E}^{\prime}$ velocities $(-0.5 \mathrm{~cm} / \mathrm{s}(0.2))$, and a greater $\mathrm{E} / \mathrm{E}^{\prime}$ ratio $(0.5(0.2))$. Additionally, left ventricular longitudinal strain in the apical two-chamber view was worse by $0.9 \%(0.3)$ in individuals with metabolic syndrome compared with those without, with a trend towards worse global longitudinal strain (GLS) (0.4 (0.2), $\mathrm{p}=0.07)$. There were no differences in left ventricular volumes, ejection fraction, or stroke volume. Moreover, there were no differences in right ventricular function measured by TAPSE and right ventricular fractional area change or in peak right atrial or right ventricular pressure gradient.

In examining the relationship between the prevalence of individual criteria for metabolic syndrome with selected measures of left ventricular structure and function, high waist circumference and elevated blood pressure were associated with multiple measures of left ventricular structure and function (table 4). Specifically, high waist circumference was associated with increased LVMI $\left(4.8 \mathrm{~g} / \mathrm{m}^{2}(1.2)\right)$, increased LVEDV (6.8 $\mathrm{mL}(1.7)$ ), increased E/E' $(0.8(0.2))$, and worse left ventricular longitudinal strain in the two-chamber view $(0.6 \%$
(0.3)). Elevated blood pressure was associated with increased LVMI $\left(3.7 \mathrm{~g} / \mathrm{m}^{2}(1.6)\right)$ and increased $\mathrm{E} / \mathrm{E}^{\prime}$ ratio $(0.7(0.02))$. Elevated triglyceride level was associated with worse left ventricular longitudinal strain in the two-chamber view $(0.6 \%(0.3))$. Neither elevated fasting glucose nor low HDL were associated with any of the selected echocardiographic measures of left ventricular structure and function.

In a secondary analysis comparing individuals with and without metabolic syndrome stratified by BMI categories, the most striking differences in cardiac structure and function was observed among normal-weight individuals. Normal-weight individuals with metabolic syndrome had decreased left ventricular systolic function and decreased myocardial deformation. Specifically, after adjusting for potential confounding variables, LVEF was reduced by $3.1 \%(0.4)$ and LVGLS was decreased by $2 \%$ (0.2). Additionally, normal weight individuals with metabolic syndrome had decreased left ventricular mass index $\left(-4.7 \mathrm{~g} / \mathrm{m}^{2}(1.1)\right)$ and increased left ventricular end diastolic volume $(-2.0 \mathrm{~mL}(0.9))$ compared with normal-weight individuals without metabolic syndrome. Of note, the sample size of normal-weight individuals with metabolic syndrome was modest $(n=22)$. In overweight individuals, metabolic syndrome was associated with increased LVEF $(0.5 \%(0.2))$ and worse LVGLS $(0.2 \%(0.1))$. Moreover, metabolic syndrome was associated with smaller left ventricular volumes (LVEDV $-3.0 \mathrm{~mL}(0.7)$; LVESV $-1.5 \mathrm{~mL}(0.3)$ ). There was no difference in cardiac structure and function observed between 
Table 3 Multivariable linear regression analyses of the association of metabolic syndrome with echocardiographic measures of cardiac structure and function in Echo-SOL

\begin{tabular}{lcccc}
\hline & $\begin{array}{l}\text { Minimally adjusted } \\
\text { beta (SE) }\end{array}$ & P values & $\begin{array}{c}\text { Adjusted } \\
\text { beta (SE) }\end{array}$ & P values \\
\hline LV mass index, g/m ${ }^{2}$ & $2.2(1.4)$ & 0.11 & $2.0(5.7)$ & 0.13 \\
\hline LV end-diastolic volume, $\mathrm{mL}$ & $1.3(2.3)$ & 0.58 & $1.2(1.9)$ & 0.52 \\
\hline LV end-systolic volume, $\mathrm{mL}$ & $0.6(1.0)$ & 0.55 & $0.6(0.9)$ & 0.5 \\
\hline LV ejection fraction, \% & $-0.1(0.4)$ & 0.78 & $-0.1(0.4)$ & 0.88 \\
\hline LV stroke volume, $\mathrm{mL}$ & $-0.5(1.4)$ & 0.71 & $-0.2(1.3)$ & 0.90 \\
\hline LV longitudinal strain (four chamber), \% & $-0.2(0.3)$ & 0.54 & $-0.3(0.3)$ & 0.39 \\
\hline LV longitudinal strain (two chamber), \% & $1.0(0.4)$ & 0.0196 & $0.9(0.3)$ & $\mathbf{0 . 0 0 9}$ \\
\hline LV global longitudinal strain (average), \% & $0.5(0.3)$ & 0.056 & $0.4(0.2)$ & 0.07 \\
\hline Medial E' velocity, cm/s & $-0.5(0.1)$ & $<0.001$ & $-0.4(0.12)$ & $\mathbf{0 . 0 0 0 2}$ \\
\hline Lateral E' velocity, cm/s & $-0.6(0.2)$ & 0.016 & $-0.5(0.2)$ & $\mathbf{0 . 0 2}$ \\
\hline E/E' & $0.5(0.2)$ & 0.04 & $0.5(0.2)$ & $\mathbf{0 . 0 1}$ \\
\hline E/A ratio & $-0.07(0.02)$ & 0.0012 & $-0.06(0.02)$ & $\mathbf{0 . 0 0 3}$ \\
\hline Isovolumic relaxation time, $\mathrm{s}$ & $0.0007(0.007)$ & 0.59 & $0.0005(0.001)$ & 0.68 \\
\hline Peak RV/RA pressure gradient, $\mathrm{mm} \mathrm{Hg}$ & $-0.6(0.7)$ & 0.39 & $-0.7(0.6)$ & 0.28 \\
\hline TAPSE, cm & $0.005(0.05)$ & 0.92 & $0.02(0.04)$ & 0.54 \\
\hline RV fractional area change, \% & $-0.01(0.009)$ & 0.12 & $-0.01(0.009)$ & 0.20 \\
\hline Left atrial volume index, mL/m ${ }^{2}$ & $-0.6(0.5)$ & 0.28 & $-0.3(0.5)$ & 0.53 \\
\hline
\end{tabular}

$E / A$, mitral inflow $E$ velocity and A velocity ratio; E/E', mitral valve E velocity and E' velocity ratio; Echo-Sol, Echocardiographic Study of Latinos; LV, left ventricle; RA, right atrium; RV, right ventricle; TAPSE, tricuspid annular plane systolic excursion.

obese individuals with and without metabolic syndrome. Interaction between metabolic syndrome with overweight and obesity was significant for medial $\mathrm{E}^{\prime}$ velocity $(\mathrm{p}=0.03)$ and peak right ventricular/right atrial pressure gradient $(\mathrm{p}=0.04)$ in which increased BMI is associated with higher medial $\mathrm{E}^{\prime}$ velocity and decreased peak right ventricular/right atrial pressure gradient in relation to metabolic syndrome. No statistically significant interaction was observed between metabolic syndrome and BMI for any other echocardiographic measures of cardiac structure and function (table 5).

In a sensitivity analysis comparing individuals with normal BMI without metabolic syndrome to obese individuals with and without metabolic syndrome, multiple differences in cardiac structure and function emerged (online supplementary table 1). Compared with individuals with normal BMI without metabolic syndrome, obese individuals with and without metabolic syndrome had increased LVEDV (7.5 mL (1.7) and 9.8 mL (1.9), respectively), increased LVESV $(5.7 \mathrm{~mL} \quad(1.3)$ and $5.0 \mathrm{~mL}$ (1.4), respectively), and increased left ventricular stroke volume $(13.0 \mathrm{~mL}(2.0)$ and $10.2 \mathrm{~mL}(0.8)$, respectively) after adjusting for potential confounders. After full adjustment, worse longitudinal strain in the two-chamber view was observed in obese individuals with metabolic syndrome $(1.3 \%(0.4))$. A similar pattern of changes in cardiac structure and function was observed in comparing normal BMI individuals without metabolic syndrome to individuals with metabolic syndrome of all BMIs (online supplementary table 1 ). In sensitivity analyses that compared normal BMI individuals without metabolic syndrome to overweight individuals, similar changes in left ventricular structure and function were observed; however, worse global longitudinal strain in the two-chamber view was observed in the overweight individuals both with and without metabolic syndrome (online supplementary table 2).

\section{DISCUSSION}

The results of this study found that within the Echo-SOL cohort of Hispanic/Latino individuals living in the USA, individuals with metabolic syndrome had worse diastolic function and worse left ventricular longitudinal strain compared with individuals without metabolic syndrome. High waist circumference, elevated blood pressure, and elevated triglycerides were associated with increased left ventricular mass, increased left ventricular size and decreased left ventricular systolic function by longitudinal strain. Left ventricular longitudinal strain and LVEF were also impaired in individuals with normal weight and metabolic syndrome.

This study represents the largest study of metabolic syndrome and cardiac structure and function as measured by echocardiography in Hispanic/Latino individuals. The results of this study support the findings of studies in other populations that found an association of metabolic syndrome with echocardiographic measures of 

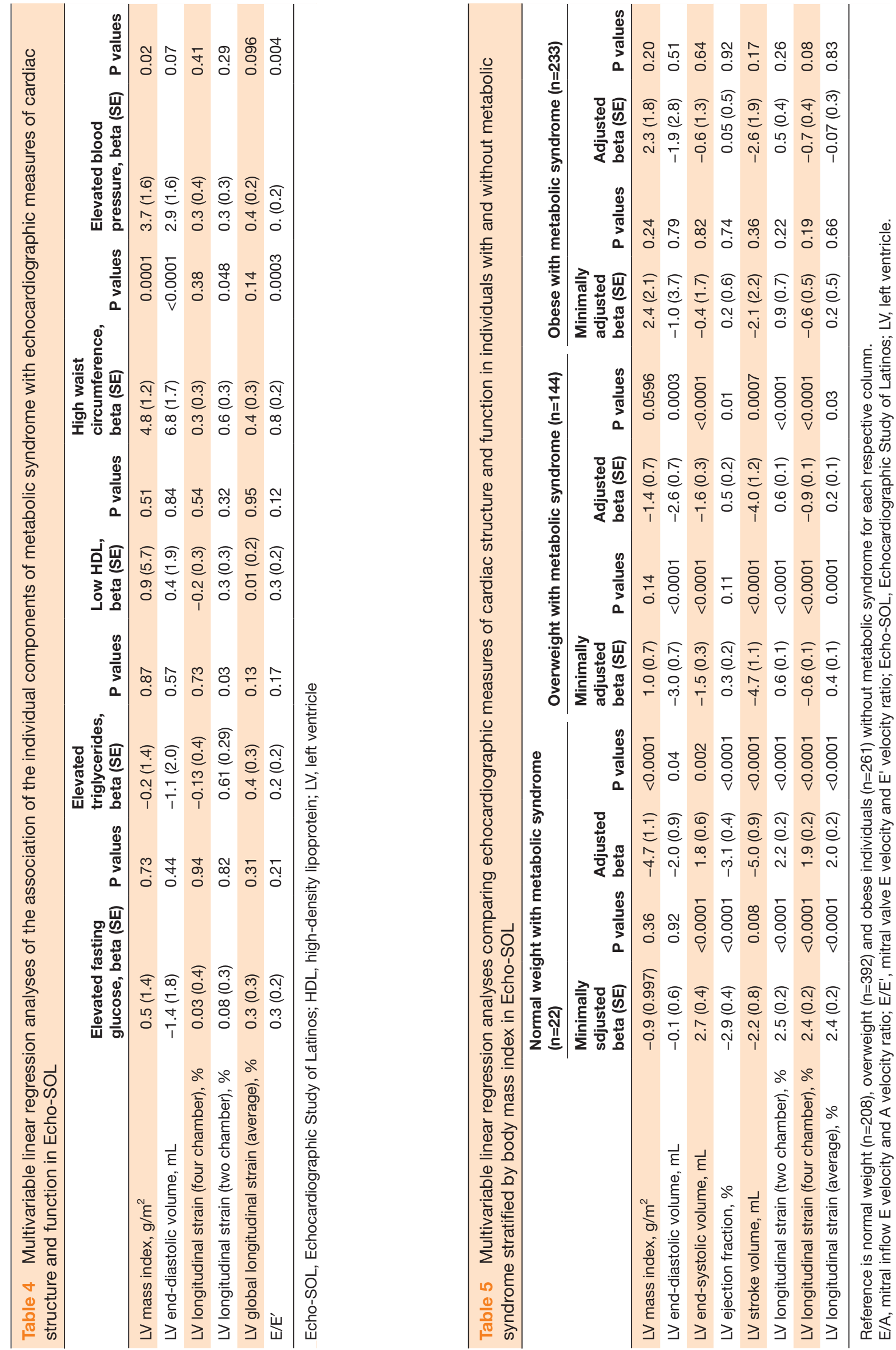
diastolic dysfunction but no difference in left ventricular mass or left ventricular volumes. ${ }^{24-30}$ Few studies have examined subclinical left ventricular systolic dysfunction by left ventricular longitudinal strain in individuals with metabolic syndrome. Prior studies include a subgroup of the Multi-Ethnic Study of Atherosclerosis cohort and a single-center study in Korea, both of which found decreased longitudinal strain in participants with metabolic syndrome. ${ }^{31} 32$ Another study observed decreased left atrial strain in individuals with metabolic syndrome, suggesting a generalized myopathic process affecting both the left ventricle and the left atrium. ${ }^{33}$ Our study did not measure left atrial strain, but did not observe any difference in left atrial size between participants with and without metabolic syndrome.

While the overall metabolic derangement that results from metabolic syndrome is likely greater than the sum of the individual criteria, we sought to understand which criteria of the metabolic syndrome were associated with changes in cardiac structure and function in individuals from Hispanic/Latino background. Surprisingly, only high waist circumference, elevated blood pressure, and elevated triglycerides were associated with changes in cardiac structure and function in this study. Additionally, high waist circumference was associated with the greatest derangement of left ventricular geometry and function, rather than hypertension. Hypertension is not only highly prevalent in this population but also is better characterized in its progression from cardiac remodeling and subclinical diastolic and systolic dysfunction to clinically overt heart failure. ${ }^{34}$ This finding is supported by prior analyses of the Northern Manhattan Study, which found that high waist circumference rather than BMI was associated with worse GLS. ${ }^{35}$ Additional studies are needed to better understand the role of abdominal obesity in cardiac remodeling and the pathogenesis of cardiac dysfunction.

The observed relationship between BMI and metabolic syndrome in relation to cardiac structure and function in this cohort of Hispanic/Latino individuals is particularly intriguing. While left ventricular longitudinal strain to some degree was worse in all weight/metabolic categories when compared with normal-weight individuals without metabolic syndrome, the greatest reduction in strain tended to be numerically higher in normal-weight individuals with metabolic syndrome. Additionally, only normal weight individuals with metabolic syndrome had a reduction in left ventricular ejection fraction compared with normal weight individuals without metabolic syndrome, suggesting a significant drop in left ventricular systolic function between the two groups. This observation is concerning in that it suggests that metabolically unhealthy individuals at a normal weight may have higher risk of heart failure, thus highlighting the fact that it might not be sufficient to focus on weight alone when counseling Hispanic/Latino patients on heart failure risk reduction. Given that the sample of normal-weight individuals with metabolic syndrome was small $(n=22)$, larger studies are necessary in order to better define the phenotype of normal-weight individuals with metabolic syndrome and the factors that make them vulnerable to subclinical reductions in left ventricular systolic function. Additionally, while the individuals in this study do not represent a heart failure population, the results of this study are reminiscent of the obesity paradox in which obese individuals with heart failure have less severe disease and better survival compared with normal-weight patients with heart failure. ${ }^{36}{ }^{37}$ While the cause of the obesity paradox continues to be debated, the results of this study suggest that the pathophysiology of non-ischemic cardiomyopathy related to cardiometabolic disease may differ in obese and normal-weight individuals.

While individuals with metabolic syndrome appear to be at increased risk for asymptomatic left ventricular systolic dysfunction, the clinical implications of this observation are less clear. Screening echocardiography for individuals with metabolic syndrome has not been addressed in the appropriate use criteria for echocardiography and is typically not performed in the absence of clinical symptoms that suggest cardiac disease. ${ }^{38}$ Once individuals with asymptomatic left ventricular systolic dysfunction are identified, the 2013 ACCF/AHA guideline for the Management of Heart Failure recommends aggressive risk factor control including evidence-based medications to treat hypertension, hyperlipidemia, and diabetes. ${ }^{39}$ Additionally, the guideline also suggests that individuals with asymptomatic reduced left ventricular ejection fraction be treated with ACE inhibitor and betablocker therapy to prevent clinical heart failure, a recommendation that is supported by evidence from clinical trials. ${ }^{40}{ }^{41}$ However, no clinical trial has specifically examined the efficacy of ACE inhibitors or beta blockers to reduce incident heart failure in individuals with asymptomatic left ventricular dysfunction and metabolic syndrome. Also, the optimal method of identifying individuals with asymptomatic left ventricular dysfunction in the setting of known cardiometabolic risk factors has not been addressed, and additional studies are needed to evaluate the yield and cost-effectiveness of any proposed screening strategy. Additionally, left ventricular systolic dysfunction has largely been defined by left ventricular ejection fraction. Better understanding of the degree to which left ventricular longitudinal strain can be used to identify individuals at risk for incident heart failure and increased mortality is needed.

This study has several limitations. First, it is a cross-sectional, observational study; therefore, determination of causality cannot be made. Second, we are unable to adjust for BMI in the linear regression analyses because BMI is highly collinear with waist circumference, which is a component of the definition of metabolic syndrome. Third, while this study is one of the largest studies of metabolic syndrome and cardiac structure and function by echocardiography in any population, the sample size is modest; thus, we are unable to perform stratified analyses by national origin in order to characterize 
the heterogeneity within Hispanic/Latinos. Within our sample, individuals with metabolic syndrome were disproportionately Cuban or Puerto Rican and lived in Miami. Thus, it would be interesting to compare cardiac structure and function in Cubans and Puerto Ricans with metabolic syndrome to other Hispanic/Latino groups. As the echocardiography examination of the entire HCHS/SOL cohort over age 45 is currently being performed, we will soon have the power to understand interactions between national background, environment, and cardiometabolic health.

\section{CONCLUSION}

In a sample of US Hispanics/Latinos, metabolic syndrome was associated with reduced left ventricular systolic and diastolic function, even in individuals with normal BMI. The results of this study suggest that strategies for cardiovascular disease and heart failure prevention in Hispanics/Latinos should include multiple indicators of cardiometabolic disease beyond BMI. The relationship between cardiometabolic risk factors and the progression of heart failure in Hispanics/Latinos requires further investigation to prioritize targets for primary prevention.

\section{Author affiliations}

${ }^{1}$ Stanford Health Care, Oakland, California, USA

${ }^{2}$ Department of Epidemiology and Prevention, Wake Forest School of Medicine,

Winston-Salem, North Carolina, USA

${ }^{3}$ Department of Psychology, Miller School of Medicine, University of Miami, Miami, Florida, USA

${ }^{4}$ Division of Cardiology, Albert Einstein College of Medicine, Bronx, New York, USA ${ }^{5}$ Department of Family and Preventive Medicine, University of California, San Diego, California, USA

${ }^{6}$ Division of Health Promotion and Behavioral Science, Graduate School of Public Health, San Diego State University, San Diego, California, USA

${ }^{7}$ Division of Cardiology, University of Illinois, Chicago, Illinois, USA

${ }^{8}$ Institute for Minority Health Research, University of Illinois, Chapel Hill, USA

${ }^{9}$ Department of Biostatistics, University of North Carolina at Chapel Hill, Chapel Hill, North Carolina, USA

Acknowledgements The authors thank the staff and participants of HCHS/SOL and Echo-SOL for their important contributions. Investigators website: http://cscc. unc.edu/hchs/.

Contributors Each author of this manuscript has made substantial contributions to the conception or design of the study; the acquisition, analysis, or interpretation of data; drafting the manuscript or critical revising of the manuscript; final approval of the version to be published; and agree to be accountable for all aspects of the work.

Funding The Hispanic Community Health Study/Study of Latinos (HCHSSOL) was performed as a collaborative study supported by contracts from the National Heart, Lung and Blood Institute (NHLBI) to the University of North Carolina (N01HC65233), University of Miami (N01-HC65234), Albert Einstein College of Medicine (N01- HC65235), Northwestern University (N01-HC65236), and San Diego State University (N01- HC65237). The following Institutes/Centers/Offices contribute to the HCHS/SOL through a transfer of funds to the National Heart, Lung and Blood Institute (NHLBI): National Institute on Minority Health and Health Disparities, National Institute on Deafness and Other Communication Disorders, National Institute of Dental and Craniofacial Research, National Institute of Diabetes and Digestive and Kidney Diseases, National Institute of Neurological Disorders and Stroke, and National Institutes of Health Institution-Office of Dietary Supplements. ECH0-SOL was supported by a grant from the NHLBI (R01 HL104199,

Epidemiological Determinants of Cardiac Structure and Function among Hispanics: Carlos J Rodriguez, MD, MPH Principal Investigator).
Competing interests None declared.

Ethics approval North Carolina.

Provenance and peer review Not commissioned; externally peer reviewed.

Data sharing statement Data from the HCHS/SOL and Echo-SOL studies can be accessed by contacting the $\mathrm{HCHS} / \mathrm{SOL}$ publications committee via the investigators website https://sites.cscc.unc.edu/hchs/.

Open access This is an open access article distributed in accordance with the Creative Commons Attribution Non Commercial (CC BY-NC 4.0) license, which permits others to distribute, remix, adapt, build upon this work non-commercially, and license their derivative works on different terms, provided the original work is properly cited, appropriate credit is given, any changes made indicated, and the use is non-commercial. See: http://creativecommons.org/licenses/by-nc/4.0/.

\section{REFERENCES}

1. Bureau UC. Population Estimates. 2014 http://factfinder.census.gov/ bkmk/table/1.0/en/PEP/2014/PEPASR6H?slice=hisp hisp!year est72014>.

2. Daviglus ML, Talavera GA, Avilés-Santa ML, et al. Prevalence of major cardiovascular risk factors and cardiovascular diseases among Hispanic/Latino individuals of diverse backgrounds in the United States. JAMA 2012;308:1775-84.

3. Dominguez K, Penman-Aguilar A, Chang MH, et al. Vital signs: leading causes of death, prevalence of diseases and risk factors, and use of health services among Hispanics in the United States 2009-2013. MMWR Morb Mortal Wkly Rep 2015;64:469-78.

4. Miller JM, Kaylor MB, Johannsson M, et al. Prevalence of metabolic syndrome and individual criterion in US adolescents: 2001-2010 National Health and Nutrition Examination Survey. Metab Syndr Relat Disord 2014;12:527-32.

5. Arguelles W, Llabre MM, Sacco RL, et al. Characterization of metabolic syndrome among diverse Hispanics/Latinos living in the United States: latent class analysis from the Hispanic Community Health Study/Study of Latinos (HCHS/SOL). Int J Cardiol 2015;184:373-9.

6. Lakka HM, Laaksonen DE, Lakka TA, et al. The metabolic syndrome and total and cardiovascular disease mortality in middle-aged men. JAMA 2002;288:2709-16.

7. Sattar N, Gaw A, Scherbakova O, et al. Metabolic syndrome with and without C-reactive protein as a predictor of coronary heart disease and diabetes in the West of Scotland Coronary Prevention Study. Circulation 2003;108:414-9.

8. Bahrami H, Bluemke DA, Kronmal R, et al. Novel metabolic risk factors for incident heart failure and their relationship with obesity: the MESA (Multi-Ethnic Study of Atherosclerosis) study. J Am Coll Cardiol 2008;51:1775-83.

9. Wang J, Sarnola K, Ruotsalainen S, et al. The metabolic syndrome predicts incident congestive heart failure: a 20 -year follow-up study of elderly Finns. Atherosclerosis 2010;210:237-42.

10. Voulgari $\mathrm{C}$, Tentolouris $\mathrm{N}$, Dilaveris $\mathrm{P}$, et al. Increased heart failure risk in normal-weight people with metabolic syndrome compared with metabolically healthy obese individuals. J Am Coll Cardiol 2011;58:1343-50.

11. Bahrami H, Kronmal R, Bluemke DA, et al. Differences in the incidence of congestive heart failure by ethnicity: the multi-ethnic study of atherosclerosis. Arch Intern Med 2008;168:2138-45.

12. Vivo RP, Krim SR, Krim NR, et al. Care and outcomes of Hispanic patients admitted with heart failure with preserved or reduced ejection fraction: findings from get with the guidelines-heart failure. Circ Heart Fail 2012;5:167-75.

13. Rodriguez CJ, Allison M, Daviglus ML, et al. Status of cardiovascular disease and stroke in Hispanics/Latinos in the United States: a science advisory from the American Heart Association. Circulation 2014;130:593-625.

14. Benjamin EJ, D'Agostino RB, Belanger AJ, et al. Left atrial size and the risk of stroke and death. The Framingham Heart Study. Circulation 1995;92:835-41.

15. Hobbs FD, Roalfe AK, Davis RC, et al. Prognosis of all-cause heart failure and borderline left ventricular systolic dysfunction: 5 year mortality follow-up of the Echocardiographic Heart of England Screening Study (ECHOES). Eur Heart J 2007;28:1128-34.

16. Armstrong AC, Jacobs DR, Gidding SS, et al. Framingham score and LV mass predict events in young adults: CARDIA study. Int J Cardiol 2014:172:350-5.

17. Bombelli M, Facchetti R, Cuspidi C, et al. Prognostic significance of left atrial enlargement in a general population: results of the PAMELA study. Hypertension 2014;64:1205-11. 
18. Yeboah J, Bluemke DA, Hundley WG, et al. Left ventricular dilation and incident congestive heart failure in asymptomatic adults without cardiovascular disease: multi-ethnic study of atherosclerosis (MESA). J Card Fail 2014;20:905-11.

19. Echouffo-Tcheugui JB, Erqou S, Butler J, et al. Assessing the risk of progression from asymptomatic left ventricular dysfunction to overt heart failure: a systematic overview and meta-analysis. JACC Heart Fail 2016;4:237-48.

20. Tsao CW, Lyass A, Larson MG, et al. Prognosis of adults with borderline left ventricular ejection fraction. JACC Heart Fail 2016;4:502-10.

21. Lavange LM, Kalsbeek WD, Sorlie PD, et al. Sample design and cohort selection in the Hispanic Community Health Study/Study of Latinos. Ann Epidemiol 2010;20:642-9.

22. Rodriguez CJ, Dharod A, Allison MA, et al. Rationale and design of the echocardiographic study of Hispanics/Latinos (ECHO-SOL). Ethn Dis 2015;25:180-6.

23. Ryan T, Berlacher K, Lindner JR, et al. COCATS 4 Task Force 5: Training in echocardiography. J Am Coll Cardiol 2015;65:1786-99.

24. Chinali M, Devereux RB, Howard BV, et al. Comparison of cardiac structure and function in American Indians with and without the metabolic syndrome (the Strong Heart Study). Am J Cardiol 2004;93:40-4.

25. Burchfiel CM, Skelton TN, Andrew ME, et al. Metabolic syndrome and echocardiographic left ventricular mass in blacks: the Atherosclerosis Risk in Communities (ARIC) Study. Circulation 2005;112:819-27.

26. Grandi AM, Maresca AM, Giudici E, et al. Metabolic syndrome and morphofunctional characteristics of the left ventricle in clinically hypertensive nondiabetic subjects. Am J Hypertens 2006;19:199-205.

27. Aijaz B, Ammar KA, Lopez-Jimenez F, et al. Abnormal cardiac structure and function in the metabolic syndrome: a populationbased study. Mayo Clin Proc 2008;83:1350-7.

28. Fontes-Carvalho R, Ladeiras-Lopes R, Bettencourt P, et al. Diastolic dysfunction in the diabetic continuum: association with insulin resistance, metabolic syndrome and type 2 diabetes. Cardiovasc Diabetol 2015;14.4.

29. Wang YC, Liang CS, Gopal DM, et al. Preclinical systolic and diastolic dysfunctions in metabolically healthy and unhealthy obese individuals. Circ Heart Fail 2015;8:897-904.

30. Ratto E, Viazzi F, Verzola D, et al. Metabolic syndrome is associated with left ventricular dilatation in primary hypertension. J Hum Hypertens 2016;30:158-63.
31. Almeida AL, Teixido-Tura G, Choi EY, et al. Metabolic syndrome, strain, and reduced myocardial function: multi-ethnic study of atherosclerosis. Arq Bras Cardiol 2014;102:327-35.

32. Bae HK, Choi HS, Sohn S, et al. Cardiovascular screening in asymptomatic adolescents with metabolic syndrome. J Cardiovasc Ultrasound 2015;23:10-19.

33. Fang NN, Sui DX, Yu JG, et al. Strain/strain rate imaging of impaired left atrial function in patients with metabolic syndrome. Hypertens Res 2015;38:758-64.

34. Schwartzkopff B, Motz W, Vogt M, et al. Heart failure on the basis of hypertension. Circulation 1993;87(5 Suppl):IV66-72.

35. Russo C, Sera F, Jin Z, et al. Abdominal adiposity, general obesity, and subclinical systolic dysfunction in the elderly: A populationbased cohort study. Eur J Heart Fail 2016;18:537-44.

36. Sharma A, Lavie CJ, Borer JS, et al. Meta-analysis of the relation of body mass index to all-cause and cardiovascular mortality and hospitalization in patients with chronic heart failure. Am J Cardiol 2015;115:1428-34

37. Oga EA, Eseyin OR. The obesity paradox and heart failure: a systematic review of a decade of evidence. J Obes 2016;2016:1-9.

38. Douglas PS, Garcia MJ, Haines DE, et al. ACCF/ASE/AHA/ASNC/ HFSA/HRS/SCAI/SCCM/SCCT/SCMR 2011 Appropriate Use Criteria for Echocardiography. A Report of the American College of Cardiology Foundation Appropriate Use Criteria Task Force, American Society of Echocardiography, American Heart Association, American Society of Nuclear Cardiology, Heart Failure Society of America, Heart Rhythm Society, Society for Cardiovascular Angiography and Interventions, Society of Critical Care Medicine, Society of Cardiovascular Computed Tomography, Society for Cardiovascular Magnetic Resonance American College of Chest Physicians. J Am Soc Echocardiogr 2011;24:229-67.

39. Yancy CW, Jessup M, Bozkurt B, et al. 2013 ACCF/AHA guideline for the management of heart failure: a report of the American College of Cardiology Foundation/American Heart Association Task Force on Practice Guidelines. J Am Coll Cardiol 2013;62:e147-239.

40. Dargie HJ. Effect of carvedilol on outcome after myocardial infarction in patients with left-ventricular dysfunction: the CAPRICORN randomised trial. Lancet 2001;357:1385-90.

41. Jong P, Yusuf S, Rousseau MF, et al. Effect of enalapril on 12-year survival and life expectancy in patients with left ventricular systolic dysfunction: a follow-up study. Lancet 2003;361:1843-8. 